\title{
Sepsis and septic shock - is a microcirculation a main player?
}

\author{
Małgorzata Lipińska-Gediga \\ Department of Anaesthesiology and Intensive Care, $4^{\text {th }}$ Military Hospital of Wroclaw, Poland
}

\begin{abstract}
Shock, defined at a cellular level, is a condition in which oxygen delivery to the cells is not sufficient to sustain cellular activity and support organ function. The central role of microcirculation in providing oxygen to the cells makes it of prime importance in determining organ function. In sepsis and septic shock, macrocirculatory alterations and microcirculatory dysfunction participate concurrently in the pathophysiology of organ failure. Haemodynamic coherence in shock is a condition in which normalization of systemic haemodynamic variables results in simultaneous amelioration in the perfusion of the microcirculation and restoration of tissue oxygenation as a final result. Septic shock is most frequently characterized by a lack of microcirculatory recruitment despite of macrocirculatory successful resuscitation. The lack of haemodynamic coherence between macrocirculation and microcirculation in septic patients results in treatment failure and increased mortality. The monitoring of microcirculation and the effects of its changes are an important area of future clinical research and treatment modification.
\end{abstract}

Key words: sepsis; microcirculation; tissue perfusion; endothelium

According to the newest definitions, sepsis should be defined as life-threatening organ dysfunction caused by a dysregulated host response to infection. For clinical operationalization, organ dysfunction may be represented by an increase in the Sequential Organ Failure Assessment (SOFA) score of 2 points or more, which is associated with an in-hospital mortality greater than $10 \%$. Septic shock should be defined as a subset of sepsis in which particularly profound circulatory, cellular and metabolic abnormalities are associated with a greater risk of mortality than with sepsis alone [1].

Sepsis and septic shock are characterized by macrocirculatory alterations such as relative hypovolaemia, a decrease in vascular tone, myocardial depression, a heterogeneous pattern of blood flow in microcirculation, as well as by the incapacity of cells to extract and adequately use oxygen. Clinical and global haemodynamic parameters show a poor correlation with the state of tissue perfusion. The microcirculatory alterations may persist after the correction of systemic hemodynamic variables [2].

\section{MICROCIRCULATION, ENDOTHELIUM, GLYCOCALYX - A PHYSIOLOGICAL POINT OF VIEW}

Microcirculation comprises a network of small blood vessels (< $100 \mu \mathrm{m}$ diameter) which comprises arterioles, capillaries and venules. It includes endothelial cells, smooth muscle cells (mostly in arterioles), red blood cells (RBC), leukocytes and platelets. Microcirculation ensures oxygen delivery to tissues, exchange of nutrients and waste products as well as modulating inflammation and coagulation. Most of these functions are controlled by endothelial cells, which regulate local blood flow, cells adhesion, permeability, as well as coagulation activation [3]. Normal microcirculation is characterized by a network of perfused capillaries with a mechanism called autoregulation, which is a regulatory mechanism allowing microcirculatory flow to stay independent of changes in systemic blood pressure. The main component of this auto-regulated system are the endothelial cells. The blood vessel lumen is lined by endothelial cells which are the primary component of the microvascular permeability barrier. Vessel integrity is influenced by the 
interaction of endothelial cells with the extracellular matrix, glycocalyx and supporting cells. The main inter-endothelial junctions are the tight junction and adherens junction. These junctions interface with the cell's cytoskeleton via specific adaptor proteins. Adherens junctions have been identified in almost all types of vascular beds, especially in the peripheral microvasculature [4]. The endothelial monolayer functions in order to control the influx and efflux of substances between the interstitium and vessel lumen. The cell-cell/matrix adhesion-based structure is semi-permeable to water and non-lipophilic molecules, providing size-and charge-selectivity for solute transport across the microvessel wall [5]. The permeability properties of the endothelial barrier are critical in the maintenance of fluid homeostasis and in the regulation of the physiological functions of tissues and organs [5]. The endothelial glycocalyx (EG) is a complex and multicomponent negatively charged layer at the luminal surface of the vascular endothelium. The EG consists of endothelial membrane-bound molecules, including glycoproteins with short branched carbohydrate side-chains and proteoglycans with long unbranched glycosaminoglycan side-chains (GAG), that provide the basis for plasma-endothelial cell interaction [6]. In and on top of this net are incorporated plasma and endothelium-derived soluble components. The composition of membrane-bound elements, as well as the composition of associated plasma proteins and soluble glycosaminoglycans, is not a static structure. Indeed, the whole endothelial surface layer is very variable, with a dynamic equilibrium between this layer of soluble components and flowing blood $[4,6,7]$.

\section{MICROCIRCULATION IN SEPSIS}

In sepsis, microcirculatory alterations are both quantitative, such as reduced vessel density and qualitative, such as altered blood flow [7]. Microcirculatory dysfunction is characterized by heterogeneous abnormalities in blood flow (slowed, intermittent, or stopped) with some capillaries being underperfused while others have normal to abnormally high blood flow. These alterations in microvascular perfusion are characterized by a decrease in vascular density together with an increased number of capillaries with stopped or intermittent flow [8]. Heterogeneity of perfusion is associated with heterogeneity in oxygenation and with altered oxygen extraction capabilities. In physiological conditions, the recruitment of microcirculation is not maximal, while it is most likely that a mild degree of heterogeneity may be observed. In response to systemic low flow, the microcirculation tends to adapt by recruiting previously unfilled capillaries and decreasing perfusion heterogeneity. When endothelial dysfunction occurs with concomitant heterogeneity, these adaptive mechanisms are lost. Tissues limit the impact of perfusion decrease by limiting the me- tabolism leading to a decrease in $\mathrm{O}_{2}$ consumption. In sepsis, as microcirculatory heterogeneity can neither be improved in response to changes in oxygen demand, nor to decreases in oxygen delivery, tissue perfusion and oxygenation are compromised [9].

In sepsis/septic shock, microcirculatory dysfunction occurs as a result of endothelial dysfunction, leukocyteendothelium interactions, coagulation and inflammatory disorders, hemorheologic abnormalities, functional shunting [3], as well as autoregulation mechanism failure [3]. The autoregulation mechanism failure occurs due to an altered expression of inducible nitric oxide synthase (iNOS) in some areas of the vascular bed. In regions where iNOS is poorly expressed, vasodilation may be limited up to the degree that the perfusion is not adequately ensured resulting in a pathological shunting of blood flow [7]. Ince [1] introduced four types of microcirculatory alterations underlying the loss of hemodynamic coherence between macro- and microcirculation. Heterogeneity in microcirculatory perfusion with obstructed capillaries next to capillaries with flowing red blood cells was classified as type 1, being typically observed in septic patients. Ince stated that hemodynamic coherence between the macrocirculation and the microcirculation is the condition in which resuscitation of systemic hemodynamics variables results in concurrent correction regional and microcirculatory perfusion and tissues oxygen delivery [10]. Loss of hemodynamic coherence is most frequently found in septic patients in whom a lack of microcirculatory recruitment is observed despite successful macrocirculatory resuscitation [10].

In a multicenter, international microcirculatory observational study carried out in 501 intensive care patients from 36 ICUs worldwide, Vellinga et al. [11] found a high heart rate as an independent risk factor for in-hospital mortality. Moreover, it was observed that if this was associated with microcirculatory alterations, then the chance of in-hospital mortality doubled.

The degree of microcirculation dysfunction is an independent predictor of survival with the proportion of perfused capillaries being the strongest predictor of outcome. Vascular density and the vascular density of perfused capillaries have been associated with outcome as well as the heterogeneity index, but not with velocity in perfused capillaries [12]. Septic patients with significant alterations in sublingual microcirculation had the worst prognosis compared with those who did not show such alterations in microcirculation [13]. Microcirculatory alterations also differ in survivors vs. non-survivors groups, rapidly improving in survivors but remaining disturbed in non-survivors [14]. De Backer et al. [15] showed a lack of correlation between arterial blood pressure and microvascular perfusion in sepsis, while also demonstrating a relationship between the pro- 


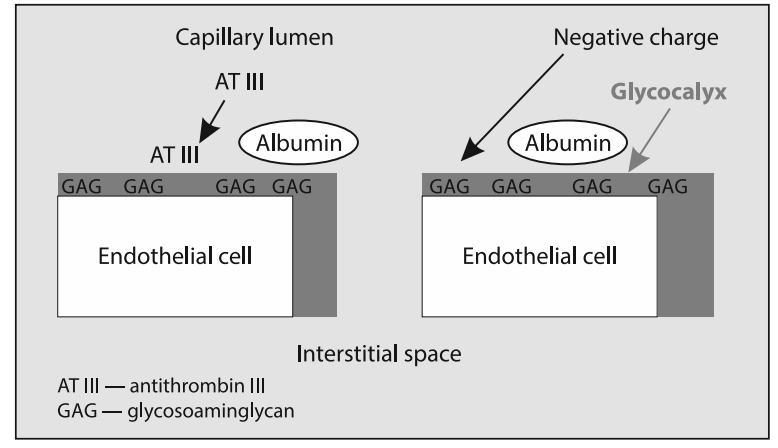

Figure 1. Endothelial glycocalyx layer in physiological states

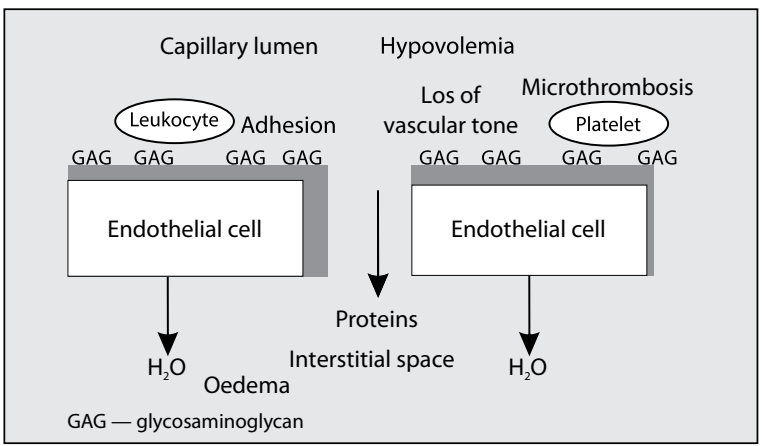

Figure 2. Glycocalyx/endothelium changes in sepsis/septic shock portion of perfused capillaries and mortality. Compared with non-infected critically ill patients and healthy volunteers, the sublingual microcirculation of patients with severe sepsis, is characterised by a significant decrease in vessel density and in proportion of perfused small vessels (48\% in septic patients compared to $90 \%$ in controls) and by increase in non-perfused, as well as in intermittently perfused vessels [15]. All these alterations are observed very early in the course of sepsis. Trzeciak et al. [16] assessed the impact of early alterations in microvascular perfusion in response to resuscitation therapies and the consequent organ dysfunction. Indeed, the authors revealed that an improvement in microvascular perfusion resulted in subsequent organ function improvement, while organ function deteriorated when microvascular perfusion failed to improve [16].

\section{ROLE OF GLYCOCALYX AND ENDOTHELIUM IN SEPSIS}

The glycocalyx plays a key role in regulating microvascular tone and endothelial permeability, maintaining an oncotic gradient across the endothelial barrier, regulating adhesion/migration of leukocytes and inhibiting intravascular thrombosis (Fig. 1).

The release of nitric oxide, contributing to the regulation of vasomotor tone and the peripheral distribution of blood flow/oxygen to tissues is a result of conformational changes in glycocalyx structure [17].

Sepsis is associated with altered micro-haemodynamics and heterogeneous local perfusion, micro-thrombosis and endothelial dysfunction, the alteration of permeability and interstitial fluid shift. Sepsis leads to the ubiquitous degradation of the glycocalyx, as well as hypovolaemia, hypoalbuminemia and oedema as a result of increased endothelial permeability. Increased sepsis, leukocyte and platelet rolling and adhesion to the endothelium impair the circulation of other cells. Red blood cells also play a role, with alterations in their deformability, the impaired release of nitric oxide
(NO) and/or the adhesion of red blood cells to the endothelium. Loss of vascular tone, the degradation of heparan sulfate leading to a shift toward a pro-coagulant state with micro-thrombosis, the production of reactive oxygen species (ROS), which disrupt microcirculatory structures with capillary obstruction by platelet/fibrin clots are changes seen in glycocalyx and endothelium function during sepsis/ septic shock (Fig. 2).

The persistence of these alterations is associated with poor clinical outcomes even if macrohaemodynamic variables have been corrected.

\section{EVALUATION OF MICROCIRCULATION}

As heterogeneous decrease in perfusion is less tolerated than a homogenously decreased perfusion, the diagnostic tool used to assess the microcirculation should detect the heterogeneity of perfusion. This is achieved with two handheld videomicroscopic imaging techniques applied at the bedside, such as OPS (orthogonal polarization spectral) and SDF (sidestream darkfield) [18]. Both techniques used the principle that if a light source is directed at a tissue, the light is reflected by the deeper layers of the tissue with transillumination of the superficial layers. As with both techniques, the selected wavelength $(530 \mathrm{~nm})$ is absorbed by the haemoglobin in red blood cells (RBC), independently of its oxygenation state, RBC are seen as black/gray points. In OPS, the applied light is polarized while the light reflected by the surface of the tissue is still polarized and is discarded by a polarizer filter [19]. The SDF technique, however, uses pulsed green light which is delivered to the tissue by multiple peripheral emitting diodes while the optics are located centrally [20]. Although both devices provide good quality images of microvascular vessels filled with RBC, SDF provides more detailed visualization of capillaries, with sharper and less granular images than OPS. These methods can be used at wide ranges of haematocrit. Both techniques allowed clinicians to assess, at the bedside, the sublingual microcir- 
culation reflecting the splanchnic microcirculation, as was demonstrated by Boerma [6, 21].There are some limitations regarding both methods. As the vascular wall cannot be visualized, only vessels containing RBC may be detected [18] while RBC that are not contained in vessels may disturb visualization of microvessels [22]. The main limitations of these methods are a restriction to semi-quantitative scoring, limited sites for investigation, as well as movement and pressure artifacts [18]. The movement artifacts may falsely interrupt the flow in some microvessels [10]. Secretions and movement artifacts may impair image quality, while the investigation of the sublingual area is only feasible in sedated or cooperative patients and is impossible in patients treated with non-invasive ventilation [23].

\section{THERAPEUTIC IMPLICATIONS}

In sepsis/septic shock concerning microvascular alterations, increasing total flow to a given organ is less important than recruiting the microcirculation. Most interventions that are currently used for their impact on systemic haemodynamics mostly influence the microcirculation to some degree. Fluids and vasoactive agents are key factors of hemodynamic resuscitation. Both Ospina-Tascon et al. and Pottecher et al. [24, 25] have demonstrated in two trials that fluids can improve microvascular perfusion, increasing the proportion of perfused capillaries and decreasing perfusion heterogeneity. In these trials the microcirculatory effects were relatively independent of the macrocirculatory effects. The microcirculatory influence of fluids seems to be mostly present in the early phase of sepsis (within 24h of diagnosis). After $48 \mathrm{~h}$, fluid administration failed to improve the microcirculation even when cardiac output was corrected [17]. Jhanji et al. [26] demonstrated that increasing MAP from 60 to $90 \mathrm{~mm} \mathrm{Hg}$ with norepinephrine infusion resulted in an increase in global $\mathrm{DO}_{2}$ but caused no change in tissue $\mathrm{pO}_{2}$ and in sublingual microcirculation. Dubin et al. [27] titrated norepinephrine to increase MAP from 65 to 75 and then to $85 \mathrm{mmHg}$, evaluating the effects on sublingual microcirculation in septic patients. The main finding was that increasing MAP failed to improve sublingual microcirculation or any other variable related to tissue oxygenation or perfusion, such as arterial lactate, anion gap, $\mathrm{pCO}_{2}$ gradients, and oxygen-derived parameters. The individual responses were strongly dependent on the basal condition of microcirculation. Although the increase in arterial pressure impaired the sublingual microcirculation in patients with close to normal microcirculation at the baseline, it was beneficial in the most severe cases [27].

The rationale for the use of vasodilators in sepsis is based on experimental and clinical findings. Vasodilators may increase the driving pressure of blood flow at the en- trance of the microcirculation and perfuse hypoxic zones. Nitroglycerin undergoes intracellular metabolism resulting in NO-mediated vasodilatation. Despite the fact that excessive NO production is believed to play an important role in sepsis-induced hypotension, it has been suggested as a therapeutic strategy in order to overcome heterogeneity in microcirculatory blood flow. Boerma et al. [28], in a double-blind placebo-controlled study, demonstrated that although sublingual microcirculatory perfusion improved significantly over time, no effect of NTG in comparison to the placebo could be shown. Various anticoagulant agents have been shown to improve the microcirculation, including activated protein C, antithrombin III, and low molecular weight heparin [7]. Levosimendan, a calcium-sensitizer drug with inotropic and vasodilator effects, has been proven useful in cardiogenic shock. In a very recent study, dobutamine at a dose of $5 \mathrm{\mu g} \mathrm{kg}^{-1} \mathrm{~min}^{-1}$ failed to improve microcirculatory blood flow [29]. In contrast, some clinical trials have shown favourable effects of levosimendan on regional and microcirculatory flow, as compared to dobutamine, in septic shock. According to a meta-analysis performed by Zangrillo in patients with severe sepsis and septic shock, levosimendan is associated with a significant reduction in mortality compared with standard inotropic therapy [30]. Moreover, Morelli et al. demonstrated that levosimendan at $0.2 \mu \mathrm{g} \mathrm{kg}^{-1} \mathrm{~min}^{-1}$ improved microcirculatory blood flow in patients with septic shock, a fact which was independent of changes in cardiac output [31]. According to Morelli, this finding suggests that effects of levosimendan at the level of the endothelium, such as the ability to decrease cytokine synthesis, plasma levels of endothelin-1, ICAM-1, VCAM-1 may have contributed to the improvement in the microcirculation $[32,33]$.

To sum up, microcirculatory alterations are associated with adverse outcomes in a manner being independent of systemic hemodynamic variables. As the effects of treatments such as fluids, vasopressors, vasodilators, anticoagulant agents are quite variable in septic patients, the monitoring of microcirculation and the effects of its changes are an important area of future clinical research and treatment modification.

\section{ACKNOWLEDGEMENTS}

1. Source of funding: none.

2. Conflict of interest: none.

\section{References:}

1. Singer $M$, Deutschman S, Seymour $\mathrm{CH}$ et al.: The Third International Consensus Definitions for Sepsis and Septic Shock (Sepsis-3). JAMA 2016; 315: 801-810. doi:10.1001/jama.2016.0287.

2. Spanos A, JhanjiS, Vivian-Smith A, Harris T, Pearse RM: Early microvascular changes in sepsis and severe sepsis. Shock 2010; 33: 387-391. doi: 10.1097/SHK.0b013e3181c6be04

3. Hernandez $G$, Bruhn A, Ince C: Microcirculation in sepsis: new perspectives. Curr Vasc Pharmacol 2013; 11: 161-169. 
4. Lipinska-Gediga M: Endothelium as a Part of Septic Multiple Organ Dysfunction Syndrome (MODS)-Is Endocan an Answer? J Clin Cell Immunol 2015; 6: 1 http://dx.doi.org/10.4172/2155-9899.1000304.

5. Steinberg $B E$, Goldenberg $N M$, Lee $W L$ : Do viral infections mimic bacterial sepsis? The role of microvascular permeability: A review of mechanisms and methods. Antiviral Res 2012; 93:2-15. doi: 10.1016/j. antiviral.2011.10.019.

6. Reitsma S, Slaaf DW, Vink H, van Zandvoort MA, oude Egbrink MG: The endothelial glycocalyx: composition, functions, and visualization. Pflugers Arch 2007; 454: 345-359. doi: 10.1007/s00424-007-0212-8.

7. Donati $A$, Domizi $R$, Damiani $E$, Adrario E, Pelaia P, Ince C: From macrohemodynamic to microcirculation. Crit Care Res Pract 2013; Article ID $892710 \mathrm{http}: / / \mathrm{dx}$. doi.org/10.1155/2013/892710.

8. De Backer D, Orbegozo CD, Donadello K, Vincent JL: Pathophysiology of microcirculatory dysfunction and the pathogenesis of septic shock. Virulence 2014; 5: 73-79 doi: 10.4161/viru.26482.

9. Marechal $X$, Favory $R$, Joulin $O$ et al.: Endothelial glycocalyx damage during endotoxemia coincides with microcirculatory dysfunction and vascular oxidative stress. Shock 2008; 29: 572-576. doi: 10.1097/ SHK.0b013e318157e926.

10. Ince $C$ : Hemodynamic coherence and the rationale for monitoring the microcirculation. Crit Care 2015; 19:S8. doi: 10.1186/cc14726.

11. Vellinga $N$, Boerma $C$, Koopmans $M$ et al.: International study on Microcirculatory Shock Occurrence in Acutely ill Patients (microSOAP). Crit Care Med 2015; 43: 48-56. doi: 10.1097/CCM.0000000000000553.

12. Edul VS, Enrico C, Laviolle B, Vazquez AR, Ince C, Dubin A: Quantitative assessment of the microcirculation in healthy volunteers and in patients with septic shock. Crit Care Med 2012; 40: 1443-1448. doi: 10.1097/ CCM.0b013e31823dae59.

13. Sakr Y,Dubois MJ, De BackerD, Creteur J, Vincent JL: Persistent microcirculatory alterations are associated with organ failure and death in patients with septic shock. Crit Care Med 2004; 32: 1825-1831.

14. Kolářová H, Ambrůzová B, Svihálková, Šindlerová L, Klinke A, Kubala L: Modulation of endothelial glycocalyx structure under inflammatory conditions. Mediators Inflamm 2014; 2014: 694312 http://dx.doi. org/10.1155/2014/694312.

15. De Backer D, Creteur J, Preiser JC, Dubois M. Vincent JL: Microvascular blood flow is altered in patients with sepsis. AJRCCM 2002; 166: 98-104.

16. Trzeciak S, McCoy JV, Phillip DR et al.: Early increases in microcirculatory perfusion during protocol-directed resuscitation are associated with reduced multiorgan failure at $24 \mathrm{~h}$ in patients with sepsis. Intensive Care Med 2008; 34: 2210-2217 doi: 10.1007/s00134-008-1193-6.

17. Chelazzi C, Villa G, Mancinelli P, De Gaudio AR, Adembri C: Glycocalyx and sepsis-induced alterations in vascular permeability. Crit Care 2015; 19 : 26. doi: 10.1186/s13054-015-0741-z.

18. De Backer D, Ospina-Tascon G, Salgado D, Favory R, Creteur J, Vincent JL: Monitoring the microcirculation in the critically ill patient: current methods and future approaches. Intensive Care Med 2010; 36: 1813-1825. doi: 10.1007/s00134-010-2005-3.

19. Groner W, Winkelman JW, Harris AG et al.: Orthogonal polarization spectral imaging: a new method for study of the microcirculation. Nat Med 1999; 5: 1209-1212.

20. Goedhart $P$, Khalilzada $M$, Bezemer $R$, Merza J, Ince C: Sidestream dark field (SDF) imaging: a novel stroboscopic LED ring-based imaging modality for clinical assessment of the microcirculation. Optics Express 2007; 15: 15101-15114.

21. Boerma EC, Mathura KR, van der Voort $P$, Spronk $P$, Ince $C$ : Quantifying bedside-derived imaging of microcirculatory abnormalities in septic patients: a prospective validation study. Crit Care 2005; 9: R601-606. doi: 10.1186/cc3809.

22. Eriksson S, Nilsson J, Sturesson Ch: Non-invasive imaging of microcirculation: a technology review. Med Devices (Auckl) 2014; 7:445-452. doi 10.2147/MDER.S51426

23. De Becker D, Donadello K: Assessment of microperfusion in sepsis. Minerva Anesthesiol 2015; 81: 533-540.

24. Ospina-Tascon G, Neves AP, Occhipinti G et al.: Effects of fluids on microvascular perfusion in patients with severe sepsis. Intensive Care Med 2010; 36: 949-955 doi: 10.1007/s00134-010-1843-3.

25. Pottecher J, Deruddre S, Teboul JL et al.: Both passive leg raising and intravascular volume expansion improve sublingual microcirculatory perfusion in severe sepsis and septic shock patients. Intensive Care Med 2010; 36: 1867-1874 doi: 10.1007/s00134-010-1966-6.

26. Jhanji S, Stirling S, Patel N, Hinds CJ, Pearse RM: The effect of increasing doses of norepinephrine on tissue oxygenation and microvascular flow in patients with septic shock. Critical Care Med 2009; 37: 1961-1966. doi: 10.1097/CCM.0b013e3181a00a1c.

27. Dubin A, Pozo MO, Casabella CA et al.: Increasing arterial blood pressure with norepinephrine does not improve microcirculatory blood flow: A prospective study. Critical Care 2009; 13: R92. doi: 10.1186/cc7922.

28. Boerma EC, Koopmans M, Konijn A et al.: Effects of nitroglycerin on sublingual microcirculatory blood flow in patients with severe sepsis/septic shock after a strict resuscitation protocol: A double-blind randomized placebo controlled trial. Crit Care Med 2010; 38: 93-100. doi: 10.1097/ CCM.0b013e3181b02fc1.

29. Hernandez G, Bruhn A, Luengo C et al.: Effects of dobutamine on systemic, regional and microcirculatory perfusion parameters in septic shock: a randomized, placebo-controlled, double-blind, crossover study. Intensive Care Med 2013; 39: 1435-1443. doi: 10.1007/s00134-013-2982-0.

30. Zangrillo A, Putzu A, Monaco F et al:. Levosimendan reduces mortality in patients with severe sepsis and septic shock: A metaanalysis of randomized trials. J Crit Care 2015; 30: 908-913. doi: 10.1016/j.jcrc.2015.05.017.

31. Morelli A, Donati A, Ertmer C et al.: Levosimendan for resuscitating the microcirculation in patients with septic shock: a randomized controlled study. Crit Care 2010; 14: R 232. doi: 10.1186/cc9387.

32. Papp Z, Édes I, Fruhwald S et al.: Levosimendan: Molecular mechanisms and clinical implications: consensus of experts on the mechanisms of action of levosimendan. Int J Cardiol 2012; 159: 82-87. doi: 10.1016/j. ijcard.2011.07.022.

33. Morelli $A$, Passariello $M$, Singer $M$ : Inotropic support in the treatment of septic myocardial dysfunction: pathophysiological implications supporting the use of levosimendan. Ann Update Int Care Emerg Med 2014. Springer International Publishing 2014; 407-419.

\section{Corresponding author:}

Małgorzata Lipińska-Gediga MD, PhD

$4^{\text {th }}$ Military Hospital of Wroclaw

Dept. of Anaesthesiology and Intensive Care

Weigla 5, 50-981 Wrocław, Poland

e-mail: starling@poczta.onet.pl

Received: 26.07.2016

Accepted: 18.09.2016 